\title{
ABC3 Consensus: Assessment by a German Group of Experts
}

\author{
Christoph Thomssen ${ }^{a}$ Doris Augustin ${ }^{b}$ Johannes Ettl ${ }^{c}$ Renate Haidinger ${ }^{d}$ \\ Hans-Joachim Lück ${ }^{\mathrm{e}}$ Diana Lüftner ${ }^{f}$ Frederik Marmég $^{g} \quad$ Norbert Marschner $^{\text {h }}$ \\ Lothar Müller $^{\mathrm{i}}$ Friedrich Overkamp $^{\mathrm{j}}$ Eugen Ruckhäberle ${ }^{\mathrm{k}}$ Marc Thill ${ }^{\mathrm{l}}$ Michael Untch $^{\mathrm{m}}$ \\ Rachel Wuerstlein ${ }^{n}$ Nadia Harbeck ${ }^{n}$
}

\author{
a University Clinic and Outpatient Clinic of Gynecology, Martin-Luther-University, Halle an der Saale, Germany; \\ ${ }^{b}$ Breast Center of Eastern Bavaria, DONAUISAR Hospital, Deggendorf, Germany; \\ ${ }^{\mathrm{C}}$ Gynecology Outpatient Clinic, Klinikum rechts der Isar, Technical University Munich, Germany; \\ dBrustkrebs Deutschland e.V., Munich, Germany; \\ e Gyneco-Oncological Practice, Hanover, Germany; \\ ${ }^{f}$ Medical Clinic focusing on Hematology, Oncology and Tumor Immunology, Charité Berlin, Benjamin Franklin Campus, Berlin, Germany; \\ ${ }^{g}$ Gyneco-Oncology Section, National Center of Tumor Diseases (NCT), Heidelberg, Germany; \\ h Joint Practice for Interdisciplinary Oncology and Hematology, Freiburg i.Br., Germany; \\ 'Onkologie UnterEms Leer-Emden-Papenburg, Leer, Germany; \\ ¡Medical Oncologist, OncoConsult.Hamburg GmbH, Hamburg, Germany; \\ kWomen's Hospital, University Hospital Düsseldorf, Germany; \\ 'Clinic for Gynecology and Obstetrics, Interdisciplinary Breast Center, Agaplesion Markus Hospital, Frankfurt/M., Germany; \\ m Gynecology Clinic, HELIOS Hospital Berlin Buch, Berlin, Germany; \\ nBreast Center, University of Munich, Germany
}

\section{Keywords}

ABC3 Consensus 2015 - Breast Cancer, metastatic .

Patient-relevant issues

\section{Summary}

The Advanced Breast Cancer Third International Consensus Conference on the diagnosis and treatment of advanced breast cancer took place in Lisbon, Portugal, on November 5-7, 2015. This year's conference (ABC3) was focused on the treatment of metastatic breast cancer (stage IV), as it was 4 years ago at the first consensus meeting ( $A B C 1)$. A matter of particular interest was the patients' perspective. Thus, patient-relevant issues were addressed by the consensus discussions, such as those on treatment goals, quality of life, care of long-term sur-

Christoph Thomssen, Diana Lüftner, Norbert Marschner, Michael Untch, Rachel Wuerstlein, and Nadia Harbeck were members of the writing committee. Renate Haidinger was a patient representative. Christoph Thomssen and Nadia Harbeck were ABC3 panel members.

The comments of the German group of experts are based on the voting results of the $\mathrm{ABC} 3$ panelists expressed on site in Lisbon, Portugal. The present manuscript mirrors the opinions of German breast cancer experts. The official ABC3 consensus will be published somewhere else under the authorship of the $\mathrm{ABC} 3$ panelists. vivors ('survivorship issues'), and coping with diseaserelated symptoms and the side effects of treatment. Further important issues on the agenda were the use of standardized instruments for the assessment of individual treatment success ('patient-reported outcome meas-

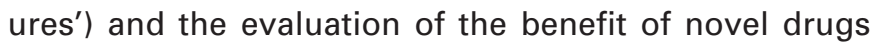
(e.g. the European Society for Medical Oncology (ESMO) Magnitude of Clinical Benefit Scale). Diagnosis and treatment of inoperable locally advanced breast cancer had already been discussed 2 years earlier at the ABC2 Consensus and were not dealt with in the framework of this year's ABC3 Consensus. With regard to country-specific peculiarities, which unavoidably found their way into the $A B C$ Consensus, a working group of German breast cancer experts commented on the voting results of the $A B C$ panelists. As for the past consensus, the group specially considered the German guidelines for the diagnosis and treatment of breast cancer (AGO (Gyneco-Oncology Working Group), S3, DGHO (German Society of Hematology and Medical Oncology)l in order to adapt the $A B C 3$ consensus for everyday therapy in Germany.

(c) 2016 S. Karger GmbH, Freiburg

\section{KARGER}

Fax +497614520714

Information@Karger.com

www.karger.com
(๑) 2016 S. Karger GmbH, Freiburg

1661-3791/16/0111-0061\$39.50/0

Accessible online at:

www.karger.com/brc
Prof. Dr. med. Christoph Thomssen

Klinik und Poliklinik für Gynäkologie

Martin-Luther-Universität Halle-Wittenberg

Ernst-Grube-Strasse 40, 06097 Halle/Saale, Germany

christoph.thomssen@ medizin.uni-halle.de 


\section{Introduction}

The organizer of the Advanced and Metastatic Breast Cancer Consensus (ABC) Conference is the European School of Oncology (ESO). The recommendations of this year's third ABC Consensus (ABC3) will be published, as usual, in the journal The Breast. The consensus is held in coordination with various international societies: the European Society for Medical Oncology (ESMO), the European Society of Breast Cancer Specialists (EUSOMA), the Federación Latinoamericana de Mastología (Latin-American Federation of Mastology (FLAM)) and the Senologic International Society (SIS). The ABC3 Consensus is also supported by the Breast Cancer Research Foundation (BCRF) and the Susan G. Komen Breast Cancer Foundation.

The goal of the ABC Consensus is the international harmonization and standardization of treatment for patients with locally advanced and/or metastatic breast cancer. The main topic of this year's $\mathrm{ABC} 3$ Consensus, just like 4 years ago during the first $\mathrm{ABC} 1$ Consensus, was metastatic breast cancer (stage IV). This year, a particular focus was on taking the patient's perspective into account. Patient-relevant issues were addressed by the consensus discussions, such as those on the aims of treatment, quality of life, care of long-term survivors ('survivorship issues'), and coping with disease-related symptoms and the side effects of treatment. Further important discussion topics were the use of standardized instruments for the assessment of individual treatment success ('patientreported outcome measures') and the evaluation of the benefit of novel drugs (e.g. the ESMO Magnitude of Clinical Benefit Scale (ESMO-MCBS)). The diagnosis and therapy of inoperable locally advanced breast cancer (stage IIIB) were discussed in the framework of the ABC2 Consensus conference, held in November 2013 in Lisbon, Portugal [1].

The ABC3 panel included 45 experts from 23 countries (table 1), among them 2 representatives from Germany (Prof. Dr. med. Nadia Harbeck and Prof. Dr. med. Christoph Thomssen) as well as 6 patient representatives and/or nursing staff. The goal is to create a valid foundation on which an individual, evidence-based therapeutic decision can be made. The statements submitted to a vote were assessed by the panelists with 'yes' (approval), 'no' (rejection), or 'abstention'. The voting results are based on the opinions of experts from different specialties who come from countries with different healthcare systems and resources. It seems therefore reasonable to discuss the voting results from a German perspective in order to substantiate them for everyday clinical practice in Germany. As for the past consensus, the group specially considered the German guidelines for the diagnosis and treatment of breast cancer (AGO (Arbeitsgemeinschaft für gynäkologische Onkologie (Gyneco-Oncology Working Group)), S3, DGHO (Deutsche Gesellschaft für Hämatologie und Medizinische Onkologie (German Society of Hematology and Medical Oncology)) [2-5] in order to adapt the ABC3 consensus for everyday therapy in Germany.

The statements discussed in the present publication of the consensus conference make up the current state of debate as of the Lisbon symposium on November 7, 2015. The final recommendations
Table 1. ABC3 Consensus panelists. (1) 
American Society of Clinical Oncology (ASCO) in the USA each developed new instruments: the ESMO-MCBS [6] and the 'ASCO Value Framework' [7], respectively. The aim is to create a standardized and balanced, objective assessment of new therapies at an international level. Based on criteria such as overall survival (OS) or progression-free survival (PFS) prolongation, toxicity, and/or quality of life, new drugs are classified as having high, medium, or only little clinical benefit. A large majority (87.5\%) of the $\mathrm{ABC} 3$ panelists welcome the new scales, particularly in order to make sure that drugs with a good benefit assessment be used and funded in countries with limited resources (level of evidence (LoE): expert opinion).

The German group of experts welcomes these scales on the grounds of possibly achieving an internationally standardized evaluation and treatment. In particular, the ESMO-MCBS, which takes into account the PFS and OS as well as the quality of life and toxicity, allows a target-oriented approach in order to expand the discussion regarding the importance of the study endpoints 'OS' versus 'PFS'. On the other hand, the ASCO scale very strongly emphasizes the cost issue, which is a crucial aspect in the American health system. The German experts specifically point out that, in Germany, neither the ESMO nor the ASCO scale has an influence on the final therapeutic decision. The physician in consultation with the patient decides on this individually.

\section{The Importance of Telemedicine}

Almost every ABC3 panelist (92.8\%) considers telemedicine to be an important treatment-supporting approach for patients living in rural or sparsely populated areas located far from oncological centers. Prerequisite is the secure use of proper information and communication technologies (LoE: expert opinion). The German group of experts agrees with this statement and adds that the cost reimbursement for this important cooperation has not yet been regulated and, in this regard, a solution still has to be found.

\section{'Patient-Reported Outcome Measures'}

A clear majority of the ABC3 panelists (87.1\%) advocate the routine (on a day-to-day basis) clinical use of validated instruments that aim at estimating the side effects of oncological treatments and disease-related complaints. These patient-reported outcome measures (PROMs) should be easy to use and clinically manageable on a day-to-day basis. This also includes an uncomplicated application, e.g. by means of cell phones. The systematic monitoring thus enabled facilitates communication between the patient and the treatment team since the undesired treatment side effects can be more reliably documented and more rapidly communicated. In addition, earlier intervention by supportive measures is possible, which means a better quality of life for the patients (LoE: IC).

From the German perspective, the routine use of these instruments is principally to be welcomed; however, it seems to be a rather unrealistic vision, considering the scarce financial resources of breast centers and of practicing doctors. For the routine use of PROMs, appropriate additional resources, e.g., for documentation and processing, must be secured.
Treatment of Long-Term Survivors ('Survivorship Issues')

Due to the improved therapeutic options, the survival period of patients with advanced and metastatic breast cancer is growing longer and longer. This poses new challenges for the day-to-day clinical practice. $95 \%$ of the $\mathrm{ABC} 3$ panelists voted for a treatment strategy that must be regularly adapted to the disease status of the patient. Herein, therapeutic effects also play a role, such as the patient's quality of life, her expectations from treatment, and her current life plans. When planning the therapy, special attention must be paid to the nursing needs of the patient, the support of her family, and her professional and social integration (LoE: expert opinion).

The German experts point out that the gain in terms of survival of patients with metastatic disease varies greatly according to subtype. Nevertheless, the demands of the ABC3 panelists are important. In Germany, there are several 'lighthouse projects' such as, e.g., the 'German Foundation for Young Adults with Cancer' [8], but a comprehensive provision of care or an adequate lobbying for these patients is still missing. The German experts add that a scientific evaluation in the frame of healthcare research is urgently required.

All ABC3 panelists (100\%) and the German experts agree that the desire of the patient to continue working during therapy must be taken into account during therapy planning, but also that an appropriate flexibility of the workplace must be available. In spite of the professional activity of the patient, the continuity of treatment must be guaranteed (LoE: expert opinion). This should, in the experts' opinion, not only be self-evident for the patients, but also society and the workplace must accept and implement this principle accordingly.

\section{Patients with Stable Disease}

More than $80 \%$ of the $\mathrm{ABC} 3$ panelists (82\%) are of the opinion that, for patients in a stable stage of their metastatic disease ('chronic condition'), oncoplastic breast reconstruction may be an option (LoE: expert opinion). The German group of experts considers the option of oncoplastic breast reconstruction in patients with metastatic disease to be feasible only in a long-term stable situation under systematic treatment. Breast reconstruction should be discussed only if the patient herself desires it. It should be considered only if the dynamics of the disease is low and a longer life expectancy is expected. In a purely palliative stage of the disease with an unstable response to treatment or extensive 'high-risk' metastatic spread, such an intervention does not make sense.

The ABC3 panelists disagreed regarding the question on whether patients with advanced disease who are stable for a long period should have regular breast imaging or whether breast imaging is only warranted in case of a suspected local or regional progression. Just over half of the ABC3 panelists (52.5\%) voted for and somewhat less than a half (47.5\%) voted against regular breast imaging (LoE: expert opinion).

From the German perspective, it is self-evident that a patient with metastatic disease must undergo physical examination at regular time intervals. In the event of a suspected local or regional pro- 
gression, breast imaging is indicated if the result of the imaging diagnosis may have clinical consequences. For patients who are free of symptoms or who do not have a suspected finding, there is no indication - from the German perspective - for regular breast imaging.

\section{Biopsy of a Metastatic Lesion}

The ABC3 panelists (97.6\%) and the German group of experts agree that a biopsy of an easily accessible metastatic lesion is important not only for histological confirmation but also for verification of the primary tumor diagnosis. Biopsy of a metastatic lesion is particularly recommended at the first appearance of metastases (LoE: 1B). The tumor biology and, in particular, the hormone receptor (HR) and human epidermal growth factor receptor 2 (HER2) status should be re-evaluated at least once in the metastatic stage, if this is clinically feasible (LoE: 1B). Depending on the localization of the metastasis (e.g. bone), technical difficulties during tissue examination should be discussed in advance with the pathologist.

The German experts add that discordant results warrant serial tissue collection and analysis during the course of the disease. Technically, in addition to a punch biopsy, a fine-needle aspiration for cytology (e.g., fluorescence in situ hybridization (FISH) in a cytological sample) may also be considered. From the German perspective, the biopsy of a metastatic lesion is also useful in case of an unexpected lack of response.

\section{Resection of the Primary Tumor}

Resection of the primary tumor in primary metastatic patients does not usually prolong survival, with the potential exception of breast cancers with merely bone metastases (LoE: 1B). Notwithstanding, breast tumor surgery can be considered in individually selected cases, in particular if an improved quality of life can thereby be expected (LoE: $2 \mathrm{~B}$ ). Just over $70 \%$ of the $\mathrm{ABC} 3$ panelists agreed on this. Prospective clinical trials are currently investigating this approach. Among others, the issues in question are as to which patients should primarily be considered for surgery and which point in time would be optimal.

The German group of experts points out that these decisions must be discussed individually with the patient, because no prolonged life expectancy has been proven to date. On the contrary, in the presence of visceral metastases, surgery can sometimes even have negative consequences. Therefore, surgery should only be considered upon request of a properly informed patient. In addition, such a patient should be stable for a long time under systemic treatment and should still have a reasonably long life expectancy and low disease dynamics. If the primary tumor is operated on, it must be removed with surrounding healthy tissue. The German experts point out that a clinically free axilla should not be treated surgically in this situation. Resection of the primary tumor is contraindicated in patients with poor response to systemic therapy and 'high-risk' metastases.

\section{Chances of Long-Term Survival?}

The German expert group agrees with the ABC3 panelists (90.6\%) that a small percentage of patients in an advanced stage of the disease have a chance of long-term survival. Usually, these are patients with oligometastatic disease or with a low metastatic burden, who respond well to systemic treatment and achieve a complete clinical remission. This group of patients should receive multimodal treatment, including local and regional therapeutic measures applied in a quasi-curative setting (LoE: expert opinion). For further validation of this approach, a prospective clinical trial is recommended.

\section{HER2-Positive Metastatic Breast Cancer}

It is not controversial that patients with HER2-positive advanced or metastatic breast cancer should be offered an antiHER2-targeted treatment as early as in the first-line therapy, if there are no contraindications (LoE: 1A).

\section{ER+/HER2+ Metastatic Disease}

Patients with HER2+ advanced breast cancer require antiHER2-targeted therapy, independent of their HR status. This applies also to the rare cases in which endocrine therapy is preferred over chemotherapy. The German group of experts and the ABC3 panelists $(72.0 \%)$ are in full agreement in this respect.

Up to now, no study has compared a first-line treatment with an endocrine agent plus an anti-HER2-targeted substance to chemotherapy plus an anti-HER2-targeted substance (LoE: 1A). The indirect comparison is in favor of chemotherapy: To date, no benefit in terms of survival could be documented for the combination of endocrine plus anti-HER2-targeted treatment, unlike for the firstline treatment with chemotherapy plus anti-HER2-targeted substances [9].

From the German perspective, the application of endocrine therapy in the first-line situation should be very restrictive. The German experts point out that, except for clinical studies, the firstline application of endocrine therapy in patients with HER2+ and estrogen receptor-positive (ER+) advanced breast cancer could be an option only if chemotherapy cannot be administered or is refused by the patient [2]. In Germany, chemotherapy plus dual HER2 blockade is the standard first-line therapy for patients with HER2-positive metastatic breast cancer, irrespective of the HR status. Currently, the DETECT V study (Chemo vs. Endo (CHEVENDO)) is running in Germany, dealing with precisely this question (i.e., endocrine therapy versus chemotherapy, each combined with dual HER2 blockade).

However, the majority of the $\mathrm{ABC} 3$ panelists (79.4\%) and the German group of experts consider endocrine therapy as an option when the chemotherapy is finished. The endocrine treatment may serve as a maintenance treatment in the event of positive ER status, with the simultaneous continuation of the anti-HER2-targeted treatment, even if this has not yet been examined in the context of randomized clinical studies (LoE: 1C).

\section{Progression under First-Line Therapy}

The vast majority (90.6\%) of the $\mathrm{ABC} 3$ panelists recommend the continuation of anti-HER2-targeted treatment in the event of 
disease progression during or after first-line treatment, and for the following lines of therapy, in addition to any cytostatic or endocrine treatment (LoE: 1B). The German experts agree on this based on the principle that the treatment of patients with HER2-positive breast cancer should always include an anti-HER2 component.

Even if it is currently not clear for how long patients with metastatic HER2-positive breast cancer require an anti-HER2-targeted treatment, further treatment with several lines of therapy is recommended. The anti-HER2 treatment should also be continued in patients with long-term remissions, as long as the therapeutic index remains positive. More than $90 \%$ of the ABC3 panelists $(92.8 \%)$ agree on this. Discontinuation of the anti-HER2 treatment after many years of a continuing and stable complete remission may be a useful option for some patients, if disease progression allows restarting the therapy at any time (LoE: expert opinion). The German experts agree, and recommend in accordance with the current AGO recommendation [2], that the anti-HER2-targeted therapy must not be interrupted if the therapeutic index remains positive, because re-induction is not always successful.

There is unanimity among all ABC3 panelists (100\%) and the German group of experts that patients who received an anti-HER2targeted treatment in the framework of a (neo)adjuvant treatment should not be excluded from clinical studies in the metastatic situation, because they continue to be candidates for anti-HER2-targeted treatment.

\section{Dual HER2 Blockade with Trastuzumab/Pertuzumab}

For patients with HER2-positive metastatic disease, in the firstline therapy, combinations of trastuzumab plus chemotherapy are superior to the combination of chemotherapy plus lapatinib, with respect to PFS and OS. This is valid independently of whether a patient was previously treated with trastuzumab in a (neo)adjuvant setting (disease-free interval (DFS) $>12$ months) or not (LoE: 1A). Almost all ABC3 panelists (95.4\%) agreed with this statement.

The German experts also agreed, in general. They added that, in Germany, dual HER2 blockade with trastuzumab/pertuzumab plus taxane is the standard first-line treatment for HER2-positive metastatic breast cancer $[2,9]$. The duration of DFS, defined as the interval between the termination of (neo)adjuvant anti-HER2 therapy and the appearance of metastases, does not play a role in the therapeutic decision (anti-HER2 treatment), in the opinion of the German experts.

The majority (85.7\%) of the ABC3 panelists also consider the dual HER2 blockade with trastuzumab/pertuzumab plus taxane as the first-line standard, but only for patients not previously treated with anti-HER2-targeted therapy. In the first-line therapy of metastatic patients with previous (neo)adjuvant anti-HER2 therapy, $75.6 \%$ of all ABC3 panelists consider the dual HER2 blockade as an 'important option'. The ABC3 panelists refer to the CLEOPATRA study, in which the dual HER2 blockade plus docetaxel in the final evaluation [8] had demonstrated a significant and clear overall median survival benefit $(\mathrm{p}<0.001)$ in comparison to trastuzumab/ docetaxel (LoE: 1A), taking into account that the majority of patients were not pretreated with HER2-targeted therapy. In addi- tion, the relatively few anti-HER2-pretreated patients were disease free for more than 12 months (LoE: 1A) [9].

The German experts agree that the differentiation made by the $\mathrm{ABC} 3$ panelists mirrors exactly the published data, and therefore the weaker recommendation for anti-HER2-pretreated patients is understandable. Nevertheless, this vote cannot be adopted as such in Germany. According to the recommendation by the AGO [2], the dual HER2 blockade plus taxane is the first-line standard in both situations. The main justification thereof is the similar relative risk reduction attributable to the dual anti-HER2 blockade, irrespective of whether the patients were previously treated with (neo) adjuvant trastuzumab or not.

In the event that chemotherapy with a taxane cannot be considered, from the German perspective, vinorelbine is an evidencebased combination partner for trastuzumab/pertuzumab [10]. The German experts believe that, in spite of an insufficient amount of data, there is also an indication for the first-line dual HER2 blockade in the event of rapid progression with a (neo)adjuvant pretreatment within 12 months (DFS $<12$ months). From the German perspective, treatment with trastuzumab emtansine (T-DM1) is an evidence-based alternative.

\section{Pertuzumab in Later Therapy Lines?}

The German group of experts agree with the majority of the $\mathrm{ABC} 3$ panelists $(86.0 \%)$ that, in view of the lack of relevant data, there is currently no indication for further (beyond the first-line therapy) treatment of HER2-positive breast cancer patients with disease progression after first-line treatment with trastuzumab/ pertuzumab plus chemotherapy (no 'pertuzumab beyond progression').

However, later application of pertuzumab/trastuzumab plus chemotherapy - beyond the first-line therapy - can be an option if the patients did not receive dual HER2 blockade in the first-line therapy (LoE: 2C). This majority vote of the ABC3 panelists (75.6\%) is supported by the German experts and would be especially beneficial for patients who could not receive pertuzumab as first-line therapy due to lack of approval.

\section{Anti-HER2-Targeted Second-Line Treatment}

Following a trastuzumab-based first-line therapy, further treatment with T-DM1 as second-line treatment is currently the most efficient option. It is superior to the combination lapatinib/capecitabine as well as to other potential therapeutic options ('treatment of physician's choice') and has a survival advantage $[11,12]$ (LoE: 1A). Almost $90 \%$ of all $\mathrm{ABC} 3$ panelists (88.0\%) agree with this statement, as does the German group of experts. The second-line treatment with T-DM1 is standard in Germany [2].

In the event of progression under trastuzumab-containing treatment, the majority of the $\mathrm{ABC} 3$ panelists (83.7\%) believe that, for some patients, the combination trastuzumab/lapatinib (without chemotherapy) could also be a useful therapeutic option (LoE: 1B). However, to date, data is lacking on the use of this combination following progression under pertuzumab/trastuzumab and/or T-DM1. - From the German perspective, it should be noted that 
the combination trastuzumab/lapatinib is approved only in steroid hormone receptor-negative, HER2-positive metastatic disease. Nevertheless, in the approval-relevant phase 3 study [13], this combination attained a median survival advantage versus the monotherapy with lapatinib for the entire collective of patients, irrespective of the HR status.

\section{Therapy Sequences and Combination Partners}

In the absence of contraindications, all patients with HER2-positive metastatic breast cancer receive an anti-HER2-targeted treatment in all treatment lines. However, the optimal therapeutic sequence of the currently available anti-HER2-targeted treatment options and the possible combination partners are unknown. They cannot be determined by vote, considering the increasing complexity of the therapy regimen and multiple combination possibilities, including those in the various pretreatments. Therefore, the $\mathrm{ABC} 3$ panel did not vote on the therapeutic sequences.

A clear majority $(86.0 \%)$ of the $\mathrm{ABC} 3$ panelists, however, voted that docetaxel and paclitaxel are the preferable combination partners for the dual HER2 blockade with trastuzumab/pertuzumab. Additional optional combination partners are vinorelbine (LoE: 1B) and nab-paclitaxel (LoE: 2B). The German experts agree with this voting result.

For countries in which pertuzumab is not available, the majority of $\mathrm{ABC} 3$ panelists $(87.8 \%)$ recommend that patients be treated in the first-line therapy with trastuzumab plus taxane or plus vinorelbin. The definitive therapeutic decision as to which kind of chemotherapy should be applied must be made individually, under consideration of the usual criteria. The German experts agree with this.

The ABC3 panelists (90.6\%) recommend the use of trastuzumab-based therapy for the later therapy lines of HER2-positive metastatic breast cancer. In addition to taxanes, possible combination partners are liposomal doxorubicin, eribulin mesylate, capecitabine, gemcitabine, or metronomic chemotherapy. The German experts agree in general, but point out that no data is currently available on the combination with gemcitabine or metronomic chemotherapy. From the German perspective, vinorelbine is an additional combination partner.

\section{HER2-Negative Metastatic Breast Cancer}

\section{ER+/HER2- Metastatic Breast Cancer}

Over $90 \%$ of the ABC3 panelists (92.6\%) and the German group of experts agreed that patients with HER2-negative (HER2-) metastatic breast cancer and positive estrogen receptor status $(\mathrm{ER}+)$ should preferably receive endocrine therapy and that this is basically also true for patients with visceral metastases. Exceptions are proven endocrine resistance or the occurrence of a potentially lifethreatening situation ('visceral crisis').

\section{Postmenopausal Patients}

For the endocrine first-line treatment of postmenopausal patients, the $\mathrm{ABC} 3$ panelists (84.0\%) recommend an aromatase in- hibitor, tamoxifen, or fulvestrant. The definitive therapeutic decision should be made depending on the adjuvant endocrine pretreatment (type and duration) and the disease-free period after termination of the adjuvant treatment (LoE: 1A). The German experts agree with this, but add that the first-line therapy should preferably include an aromatase inhibitor or fulvestrant $(500 \mathrm{mg}$ ).

Endocrine combination therapy does not play an important role in Germany. Therefore, the German experts agree with the slight absolute majority vote (53.4\%) of the ABC3 panelists who advocated not to use combined endocrine first-line treatment with a non-steroidal aromatase inhibitor plus fulvestrant in postmenopausal patients without prior adjuvant endocrine treatment. This vote was made against the background of differing results of 2 randomized phase 3 studies $[14,15]$ on ER+/HER2- postmenopausal patients with metastatic breast cancer.

In the Southwest Oncology Group (SWOG) study [14], the first-line treatment consisting of non-steroidal aromatase inhibitors plus fulvestrant had a significant advantage in terms of PFS and OS compared with the aromatase inhibitor-only treatment. In the FACT study [15] with a very similar design, no advantage could be shown. A subgroup analysis demonstrated that, in the SWOG study, the advantage could be demonstrated only in patients with no adjuvant endocrine (tamoxifen) pretreatment and with a very long DFS ( $\geq 10$ years).

The German experts point out that, in Germany, most patients with ER+/HER2- breast cancer receive adjuvant endocrine pretreatment, so that this question is irrelevant in day-to-day clinical practice. The negative vote is explained by the German experts by the fact that, in the design of the SWOG study [14], a control arm with fulvestrant $(500 \mathrm{mg}$ ) as monotherapy was missing, which was also pointed out by the SWOG study authors themselves. Thus, it is difficult to estimate the position of the combination (of non-steroidal aromatase inhibitors/fulvestrant 250). It must also be taken into account that the benefit of the aromatase inhibitors/fulvestrant combination in this subgroup of patients pretreated with tamoxifen was only small.

For postmenopausal patients with ER+/HER2- metastatic breast cancer who experience disease progression under a non-steroidal aromatase inhibitor, an option exists of further treatment with an aromatase inhibitor in combination with the mammalian target of rapamycin (mTOR) inhibitor everolimus. This therapy approach enables significant PFS extension $(p<0.001)$ and a numerical median survival advantage of almost 5 months [16]. The German experts agree with this majority vote (84.6\%) of the ABC3 panelists. There is agreement that the therapeutic decision must be made individually, considering the increased toxicity induced by everolimus (LoE: 1B). There is currently no predictive biomarker for identifying patients who will benefit from the addition of an mTOR inhibitor.

In addition, the German experts add that the combination of exemestane/everolimus and fulvestrant $(500 \mathrm{mg})$ constitute valid first-line options for postmenopausal patients with adjuvant endocrine pretreatment. The indication for this combination should be based mainly on the disease dynamics, taking into account the ex- 
pected side effects and the wishes of the informed patient. From the German perspective, the patient's age as such does not play a role in the therapeutic decision; the data of the non-interventional BRAWO study did not show any association between age and side effects [17].

\section{Significance of Palbociclib}

A potential new therapeutic option for the first-line treatment of ER+/HER2- postmenopausal patients with metastatic breast cancer is the cyclin-dependent kinase (CDK)4/6 inhibitor palbociclib, which demonstrated an impressive PFS advantage in combination with the aromatase inhibitor letrozole in a randomized phase 2 study versus the aromatase inhibitor alone (HR 0.488; $\mathrm{p}=$ $0.0004)$ [18]. Just over half (51.1\%) of all ABC3 panelists voted that the phase 2 data must still be confirmed by a phase 3 study [19] before the combination of palbociclib/aromatase inhibitor can be classified as recommended therapy option. Almost $40 \%$ of the $\mathrm{ABC} 3$ panelists (39.5\%) voted against this and were of the opinion that the phase 2 data already justify clinical use.

However, beyond the first-line treatment, a clear majority of $85.7 \%$ of the $\mathrm{ABC} 3$ panelists consider the combination of palbociclib/fulvestrant as an option for pre-, peri-, and postmenopausal patients with ER+/HER2- metastatic breast cancer. The panelists point out the significant PFS advantage shown in the interim analysis of the PALOMA-3 study with the approximate median value of 5 months (HR 0.422; p < 0.000001) and improved quality of life [20]. Pre- and perimenopausal patients additionally need a luteinizing hormone-releasing hormone (LHRH) agonist (LoE: 1B). Again, for palbociclib, there is no predictive biomarker.

The German experts point out that palbociclib has not yet been approved in Germany. The drug is registered in the USA, and German and/or European approval is expected in the course of 2016. In addition, phase 3 data is still missing for the palbociclib/aromatase inhibitor combination. In Germany a phase 3/4 intergroup study on the use of palbociclib is currently in preparation.

\section{Endocrine Treatment Sequences}

The optimal endocrine therapy sequence following an endocrine first-line treatment cannot be defined as it depends on each previously administered treatment. More than $90 \%$ of the $\mathrm{ABC} 3$ panelists (92.5\%) consider the administration of aromatase inhibitors, tamoxifen, fulvestrant/palbociclib, aromatase inhibitors/everolimus, tamoxifen/everolimus, fulvestrant alone, and megestrole acetate and estradiole as possible therapeutic options (LoE: 1A). Due to insufficient study data either comparing combination therapies consisting of an endocrine plus a biological substance or in comparison with monochemotherapy (e.g. capecitabine), no further evaluation is possible. We must wait for the results of the ongoing studies.

The German experts agree in general, but point out the still pending approval of palbociclib and add that, in Germany, gestagens and estradiol are currently used only in individual cases and that in case of fulvestrant the approved 500-mg dose must be used. From the German prospective, a well-tolerated chemotherapy can also be considered as an alternative to endocrine third-line therapy.

\section{Premenopausal Patients}

Almost all ABC3 panelists (93.0\%) voted for the statement that, for premenopausal patients with ER+/HER2- metastatic breast cancer who should receive endocrine therapy, ovarian suppression or ablation combined with an additional endocrine substance is the preferred therapeutic option (LoE: 1B). The ovarian ablation via laparoscopic bilateral oophorectomy guarantees adequate estrogen suppression including contraception, prevents potential initial flare phenomena which can occur under LHRH agonists and possibly also increases the possibility of participating in clinical studies (LoE: expert opinion). In addition, $90.6 \%$ of all $\mathrm{ABC} 3$ panelists believe that ovarian irradiation is also a possibility to achieve permanent ovarian ablation.

The German group of experts agrees with these votes. They point out that, in premenopausal patients, the additional ovarian suppression is - due to published data - a standard measure accompanying any endocrine treatment [21]. Ovarian suppression can be achieved either pharmacologically by gonadotropin-releasing hormone $(\mathrm{GnRH})$ analogues or surgically by bilateral oophorectomy. Radiation therapy should only be used if contraindications against both these options are present (such as coagulation disorders or other conditions that do not allow surgery). One should be aware that, in case of irradiation, complete endocrine ablation is only achieved with a substantial delay.

The ABC3 panelists (95.2\%) voted for the use of either an aromatase inhibitor or tamoxifen as an additional endocrine therapy for premenopausal patients, depending on the kind and duration of the adjuvant endocrine pretreatment. For pharmacological reasons, an aromatase inhibitor absolutely requires additional ovarian suppression or ablation (LoE: 1B). This is currently also valid for fulvestrant, which in the opinion of the panelists can also be considered an option (LoE: 1C). The German experts agree with this.

\section{Triple-Negative Advanced Breast Cancer}

The ABC3 panelists (97.7\%) and the group of German experts agree that, if other data is missing, the same chemotherapy recommendations are valid for patients with advanced, non-BRCA-mutated, triple-negative breast cancer (TNBC) as for patients with HER2-negative disease (LoE: 1A). Irrespective of the BRCA status, a clear majority of the ABC3 panelists (90.6\%) evaluated carboplatin treatment as an important therapeutic option for TNBC patients pretreated with anthracyclines and taxanes in the (neo)adjuvant setting. Carboplatin has a comparable efficacy to docetaxel but with a more favorable side effect profile (LoE: 1A). The German experts also agree with this.

\section{Additional Statements}

\section{Metronomic Chemotherapy}

The German experts agree with the majority of the $\mathrm{ABC} 3$ panelists $(88.3 \%)$ that metronomic chemotherapy may constitute a reasonable therapy for patients without increased remission pressure who do not require rapid tumor regression (LoE: 1B). The 
combination of low-dose cyclophosphamide and methotrexate (CM) is a well-investigated regimen [22]. Other regimens currently evaluated for their metronomic therapeutic concepts are, e.g., those with capecitabine and oral vinorelbine. However, results of randomized studies comparing metronomic chemotherapy concepts with conventional dose regimens are still lacking. The ABC3 panelists and the German experts agree that such studies are necessary.

\section{Re-Induction of Anthracyclines}

Re-induction of anthracyclines in the metastatic situation is an evidence-based option for HER2-negative breast cancer patients who were previously treated with anthracyclines as adjuvant. Almost all ABC3 (93.1\%) panelists supported this statement. Prerequisites are that the cumulative total dose of anthracyclines has not yet been reached and that there are no cardiac contraindications. Ideally, the patient should be disease free for at least 1 year after the adjuvant treatment. The German experts agree with this majority vote of the panelists and added that, for the rechallenge, anthracycline schemes with good cardiac tolerability would be particularly suitable, such as weekly anthracycline administration or pegylated liposomal formulations. Such a regimen can be an option even if the cumulative total dose has been reached.

\section{BRCA-Mutated Metastatic Breast Cancer}

The German experts agree with the majority vote of the $\mathrm{ABC} 3$ panelists (86.3\%), namely that platinum-based regimens are a preferable therapeutic option for metastatic patients with BRCA-mutated TNBC or endocrine-resistant breast cancer, if these women were already previously treated with anthracyclines or taxanes in the adjuvant or the metastatic situation. Additional conditions are that the patients have not yet received any platinum and that they cannot be included in a relevant controlled clinical study (LoE: 1A). - The ABC3 panelists (90.6\%) and the German experts point out that BRCA germline testing should be performed in TNBC patients with negative family history, if any clinical consequences could result from this.

\section{Bone Metastases}

Also concerning the application of anti-resorption substances ('bone-modifying agents') such as bisphosphonates and denosumab, there is agreement between the German experts and the majority of the $\mathrm{ABC} 3$ panelists (95.4\%): There is an indication for their use as a routine addition to further oncological treatment in patients with bone metastases (LoE: 1A). According to an ABC3 vote, the application of zoledronic acid every 3 months is not inferior to the standard monthly dosing (LoE: 1B). The German experts restrict this statement and maintain that a once-in-3-months dose can only be considered after a stable situation has been reached under the monthly standard dose. If there are no contraindications, an additional supplementation with calcium and vitamin D should definitely be recommended, and supplementation with denosumab is obligatory (LoE: 1C).

\section{Brain Metastases in HER2-Positive Disease}

More than $90 \%$ of the ABC3 panelists (95.2\%) voted for the unchanged continuation of systemic treatment in patients with HER2-positive breast cancer with brain metastases, as long as the extracranial disease is stable (LoE: 1C). For patients in whom the brain metastases are the only clinical localization of metastases, it is unclear - according to the vote of the ABC3 panelists (83.3\%) whether chemotherapy in addition to the local measures can substantially improve the course of the disease. However, it is recommended to restart an anti-HER2-targeted therapy (trastuzumab) if this was stopped in the meantime (LoE: 1C). The German experts agree with this.

\section{'Next-Generation Sequencing'}

The ABC3 panelists (94.4\%) and the German experts agree that data obtained from genomic testing by 'next-generation sequencing' (NGS) in order to detect potential molecular alterations regarding therapy and course of disease in patients with advanced breast cancer has not yet been adequately validated to allow clinical decisions to be based thereon. Results from clinical studies documenting an advantage of therapeutic decisions based on NGS are still missing, and therefore this procedure must currently be classified as investigational.

\section{Important Definitions}

\section{Oligometastatic Disease}

A majority of $77.7 \%$ of all $\mathrm{ABC} 3$ panelists define an oligometastatic breast cancer as a disease with low metastatic tumor burden and limited number of metastatic lesions. The latter means, according to the $\mathrm{ABC} 3$ vote, that a maximum of 5 lesions are present, which do not necessarily have to be in the same body organ. The German experts do not agree with this last definition: From the German perspective, oligometastatic disease is defined as a limited number of metastases in one body organ. There is unanimity that local measures are an important potential therapy option in oligometastatic disease; the aim of the therapy is complete clinical remission.

\section{'Multiple Chronic Conditions'}

All ABC3 panelists (100\%) define patients with 'multiple chronic conditions' (MCCs) as patients who have multiple (clinically relevant) comorbidities, e.g. cardiac diseases, reduced renal and/or hepatic function, or an autoimmune disease. MCC patients require close monitoring and special individual therapy concepts. It is difficult to define general therapy recommendations for the management of these patients ('difficult to account for all of the possible extrapolations to develop specific recommendations for care').

\section{Supportive and Palliative Treatment}

The question of supportive therapy and palliative treatment of patients with advanced breast cancer was not publicly voted on at 
the $\mathrm{ABC} 3$ meeting so that no comment was made from the German perspective.

\section{ABC3 - A Forum for Patient Advocates}

During the ABC3 conference, the representatives of international patient support groups also held discussions. Overall, 66 representatives from Europe, Asia, the Middle East, Africa, Australia, North, South and Central America were present. They presented the results of their work in the plenary session of the ABC3 conference on November 7, 2015. In a moving speech by Shirley A. Mertz, a patient advocate from the USA, the support groups expressed their gratitude to the physicians for their on-duty commitment to patients and emphasized how important this commitment is for each individual patient. Mertz: 'Your work affects how long we live and the quality of life we have!'

The advocacy groups also expressed their gratitude for the possibility to take part in the $\mathrm{ABC}$ conference and for the opportunity of international exchange between themselves and the physicians. This is particularly important considering the complexity of the disease. The support groups invited the scientists, caregivers, the industry, the worldwide advocacy groups, and the representatives of the media to establish a cooperation aimed at further improving the prognosis of patients with metastatic breast cancer.

Four key goals were defined for the near future:

- Creation of an 'ABC Global Advocate Community'. Its goal would be the development of a common network to exchange experiences and to promptly discuss and implement common goals and strategies.

- In close relationship with this, initiation of an 'Alliance' of regular dialog between the support groups, for example by the use of webinars. The focus of this 'Alliance' should be therapeutic recommendations and aspects of quality of life.

- The educational work with metastatic breast cancer patients regarding the disease itself but also the therapeutic options and support group possibilities should be further coordinated and improved. This also includes translating the $\mathrm{ABC} 3$ consensus into the languages spoken in the individual countries.

- In the future, the representatives of the advocacy groups also want to meet each year in person, in order to establish an open path of discussion and exchange of opinions for advancing the required goals.

\section{Conclusions and Prospects}

The $\mathrm{ABC}$ consensus again provided a forum for an informative discussion on the most recent developments related to advanced breast cancer. The $\mathrm{ABC}$ meeting is, in this respect, the consequent addition to the St. Gallen consensus conference on early breast cancer. The next ABC4 consensus conference will be held on November 9-11, 2017. The next AGO 'State-of-the-Art Meeting', during which the annually updated AGO-Mamma guidelines for the diagnosis and therapy of breast cancer will be presented, will be held on March 5, 2016.

\section{Acknowledgements}

The meeting of the German experts in Lisbon was supported and organized by MEDUCATION Institut GmbH. Financial support, without restrictions regarding the contents, was granted by Celgene $\mathrm{GmbH}$. The authors would like to thank Birgit-Kristin Pohlmann, Nordkirchen, for her editorial support during the creation of the manuscript. The final approval of the content of this manuscript is the sole responsibility of the authors.

\section{Disclosure Statement}

C.T. received honoraria from Amgen, AstraZeneca, Celgene, Genomic Health, NanoString, Novartis, Pfizer, Roche, and Teva. D.A. has no conflict of interest. J.E. received honoraria from Celgene, Eisai, GSK, Janssen, Novartis, Pfizer, Roche; a research grant from Celgene; travel grants from Celgene, GSK, Novartis, Pfizer, and Roche. R.H. has no conflict of interest. H.-J. Lück received honoraria from Roche, Novartis, Eisai, and Celgene. D.L. received honoraria from Roche, Amgen, Celgene, Novartis, and Pfizer. F.M. received honoraria from Amgen, Pfizer, Novartis, Roche, AstraZeneca, Genomic Health, and PharmaMar. N.M. has no conflict of interest. L.M. has no conflict of interest. F.O. has no conflict of interest. E.R. received honoraria from Amgen, Celgene, JanssenCilag, Novartis, Pierre Fabre, and Roche. M.T. received honoraria from Roche, Celgene, Novartis, Genomic Health, AstraZeneca, Teva, Myriad, Boehringer, and Amgen. M.U. has no conflict of interest. R.W. received honoraria from Celgene, Pfizer, Novartis, Roche, Eisai, AstraZeneca, Amgen, MSD, and Pierre Fabre. N.H. received honoraria from Celgene, Genomic Health, NanoString, Novartis, and Roche.

\section{References}

1 Cardoso F, Costa A, Norton L, et al.: ESO-ESMO 2nd international consensus guidelines for advanced breast cancer (ABC2). Breast 2014;23:489-502.

2 www.ago-online.de [last accessed November 15, 2015].

3 Liedtke C, Thill M, Hanf V, Schütz F: AGO recommendations for diagnosis and treatment of patients with advanced and metastatic breast cancer: update 2015. Breast Care (Basel) 2015;10:199-205.

4 www.awmf.org [last accessed November 15, 2015]

5 www.dgho.de/Onkopedia [last accessed November 24, 2015].
6 Cherny NI, Sullivan R, Dafni U, et al.: A standardised, generic, validated approach to stratify the magnitude of clinical benefit that can be anticipated from anticancer therapies: the European Society for Medical Oncology Magnitude of Clinical Benefit Scale (ESMOMCBS). Ann Oncol 2015;26:1547-1573.

7 Schnipper LE, Davidson NE, Wollins DS, et al.: American Society of Clinical Oncology statement: a conceptual framework to assess the value of cancer treatment options. J Clin Oncol 2015;33:2563-2577.

8 www.junge-erwachsene-mit-krebs.de.
9 Swain S, Baselga J, Kim S-B, et al.: Pertuzumab, trastuzumab and docetaxel in HER2-positive metastatic breast cancer. N Engl J Med 2015;372:724-734.

10 Andersson M, Lopez-Vega JM, Petit T, et al.: The coadministration of pertuzumab and trastuzumab as a single infusion, followed by vinorelbine in first-line treatment of HER2-positive locally advanced or metastatic breast cancer patients: VELVET study interim analysis. J Clin Oncol 2015;(Proc ASCO):abstr 586.

11 Verma S, Miles D, Gianni L, et al.: Trastuzumab emtansine for HER2-positive advanced breast cancer. N Engl J Med 2012;367:1783-1791. 
12 Wildiers H, Kim S-B, Gonzalez-Martin A, et al.: T-DM1 for HER2-positive MBC: primary results from TH3RESA, a phase 3 study of T-DM1 vs. treatment of physician's choice. Proc ECC-ESMO 2013;abstr LBA 15

13 Blackwell KL, Burstein HJ, Storniolo AM, et al.: Overall survival benefit with lapatinib in combination with trastuzumab for patients with human epidermal growth factor receptor 2-positive metastatic breast cancer: final results from the EGF104900 study. J Clin Oncol 2012;30:2585-2592.

14 Mehta RS, Barlow WE, Albain KS, et al.: Combination anastrozole and fulvestrant in metastatic breast cancer. N Engl J Med 2012;367:435-444.

15 Bergh J, Jönsson PE, Lidbrink EK, et al.: FACT: an open-label randomized phase III-study with fulvestrant and anastrozole in combination compared with anastrozol alone as first-line therapy for patients with receptor-positive postmenopausal breast cancer. J Clin Oncol 2012;30:1919-1925.
16 Baselga J, Campone M, Piccart M, et al.: Everolimus in postmenopausal hormone-receptor-positive advanced breast cancer. N Engl J Med 2012;366:520-529.

17 Tesch H, Grischke E, Fasching P, et al.: Results of the 3rd interims analysis of BRAWO - breast cancer treatment with everolimus and exemestane for ER+ women. Oncol Res Treat 2015;38(suppl):252 (P823).

18 Finn RS, Crown JP, Lang I, et al.: Final results of a randomized phase II study of PD 0332991, a cyclin-dependent kinase-4/6 inhibitor, in combination with letrozole vs letrozole alone for first-line treatment of ER+/HER2 - advanced breast cancer (PALOMA-1; TRIO-18). San Diego, AACR Annual Meeting 2014. Cancer Res 2014;74:CT101.

19 ClinicalTrials.gov: NCT01740427. https://clinicaltrials. gov.

20 Turner N, Ro J, André F, et al.: Palbociclib in hormone-receptor-positive advanced breast cancer. N Engl J Med 2015;373:209-219.
21 Klijn JG, Blamey RW, Boccardo F, et al.: Combined tamoxifen and luteinizing hormone-releasing hormone (LHRH) agonist versus LHRH agonist alone in premenopausal advanced breast cancer. A meta-analysis of four randomized trials. J Clin Oncol 2001;19:343353.

22 Curigliano G: Phase I dose-finding study of the gamma secretase inhibitor PF-03084014 (PF-4014) in combination with docetaxel in patients with advanced triplenegative breast cancer. J Clin Oncol 2015;(Proc ASCO): abstr 1068. 